\title{
L'éducation plurilingue et interculturelle au regard des conceptions morales des enseignants de langue-culture en contexte multiculturel
}

\section{Emmanuel Antier}

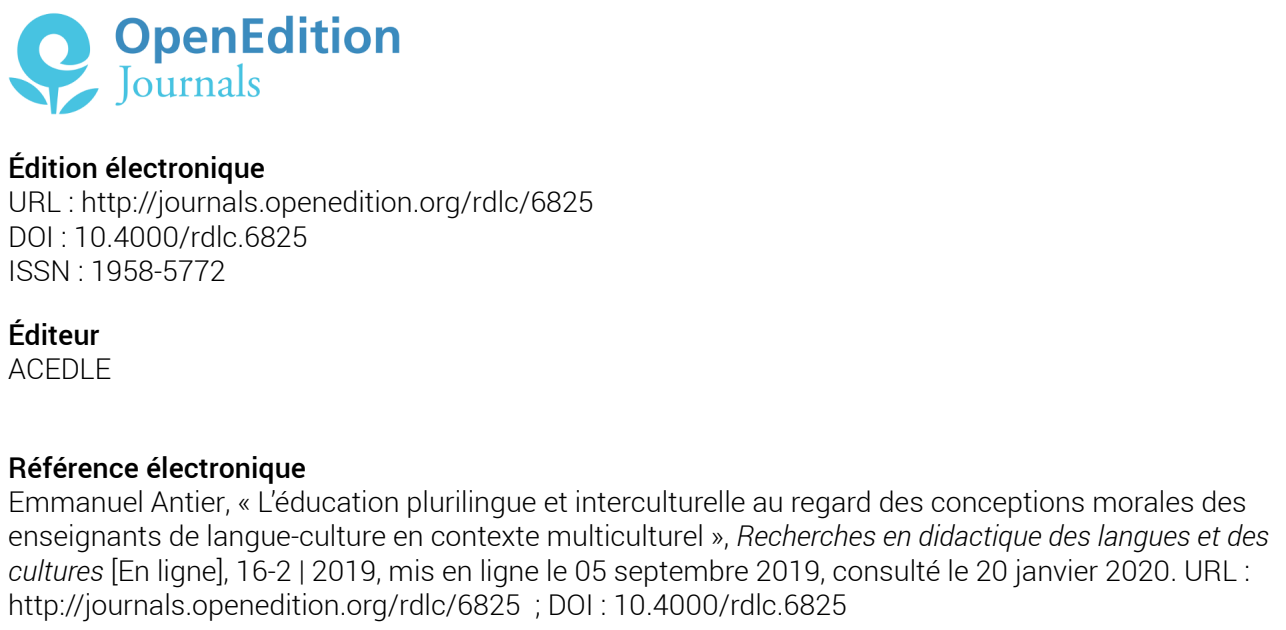

Ce document a été généré automatiquement le 20 janvier 2020.

\section{(c) (1) $\odot$}

Recherches en didactique des langues et des cultures is licensed under a Creative Commons AttributionNonCommercial-NoDerivatives 4.0 International License 


\title{
L'éducation plurilingue et interculturelle au regard des conceptions morales des enseignants de langue-culture en contexte multiculturel
}

\author{
Emmanuel Antier
}

\section{Introduction}

1 Le recours à la distinction proposée par Ogien (2007) entre le maximalisme et le minimalisme nous a permis de mettre en évidence le caractère maximaliste de certains concepts et discours en didactique des langues-cultures (Antier, 2017). Nous avons vu, notamment, que la notion de savoir-être renvoie à l'idée d'un perfectionnisme moral caractéristique des théories maximalistes. Plus généralement, il nous est apparu que l'éducation plurilingue et interculturelle prônée par le Conseil de l'Europe, en ce qu'elle tend à promouvoir une conception particulière du bien personnel, relève d'une vision maximaliste de la morale. Le passage suivant du CECR (2001) nous servira à illustrer ici ce constat :

\begin{abstract}
Dans une approche interculturelle, un objectif essentiel de l'enseignement des langues est de favoriser le développement harmonieux de la personnalité de l'apprenant et de son identité en réponse à l'expérience enrichissante de l'altérité en matière de langue et de culture. Il revient aux enseignants et aux apprenants eux-mêmes de construire une personnalité saine et équilibrée à partir des éléments variés qui la composeront. (p. 9, nous soulignons)
\end{abstract}

2 Dans cet article, nous nous proposons de faire porter l'analyse sur les conceptions morales des enseignants de langue-culture. Sont-elles maximalistes ou minimalistes? Pour le formuler autrement: les enseignants considèrent-ils, par exemple, qu'ils doivent perfectionner la personnalité de leurs apprenants ou, au contraire, rester 
neutres sur la conception du bien personnel. Quelle place accordent-ils au savoir-être dans leur enseignement? Se donnent-ils pour objectif, par exemple, et pour citer des savoir-être listés dans le CARAP (Conseil de l'Europe, 2013), de développer chez leurs apprenants une «empathie envers l'altérité » (p. 40) ou un « respect de la diversité linguistique et culturelle » (p. 41) ?

3 Afin d'apporter des éléments de réponse à ce questionnement, nous avons mené une enquête par questionnaires auprès d'enseignants de langue-culture dans un contexte multiculturel, c'est-à-dire un contexte où cohabitent plusieurs cultures éducatives, plusieurs conceptions de la relation éducative et de l'acte éducatif lui-même. À travers cette enquête, nous chercherons à tester l'hypothèse selon laquelle les enseignants en contexte multiculturel mobilisent une conception plutôt minimaliste de la morale. Si notre hypothèse est vérifiée, elle pourrait contribuer à contester la portée universaliste de l'éducation plurilingue et interculturelle, ainsi que, plus généralement, l'idée d'une éducation basée sur l'enseignement de savoir-être.

4 Mais avant de présenter les résultats de cette enquête (section 5), et de les discuter (section 6), il convient préalablement de préciser notre cadre théorique (section 2), de recenser les études empiriques menées sur cette thématique (section 3), et de rendre compte de notre méthodologie de recherche (section 4).

\section{Cadre théorique}

5 Le cadre théorique de notre étude s'appuie sur la philosophie morale contemporaine, et plus précisément sur la distinction entre le minimalisme et le maximalisme, distinction formalisée notamment par Ogien (2007). Selon lui, l'histoire de l'éthique contemporaine peut en effet se résumer à cette opposition binaire. Qu'entend-il précisément par ces mots? Les lignes d'ouverture de L'éthique aujourd'hui nous offrent un premier éclaircissement :

Imaginez un monde dans lequel on pourrait vous juger « immoral », non seulement pour vos actions, mais aussi pour vos pensées, vos désirs, vos fantasmes ou vos traits de « caractère ». Non seulement pour ce que vous faites aux autres, mais aussi pour ce que vous vous faites à vous-même. Non seulement pour ce que vous faites délibérément, en toute connaissance de cause, mais aussi pour ce qui vous arrive un peu par hasard. [...]. J'appelle « maximaliste » un tel monde moral et, par contraste, "minimaliste» des mondes moraux moins envahissants, dans lesquels, par exemple, toute l'éthique se résume au souci d'éviter de nuire délibérément à autrui. (2007:11-12)

6 Ainsi, pour Ogien, l'opposition classique entre morales kantienne et aristotélicienne apparaît comme secondaire. Ce qui compte, selon lui, c'est l'extension que les différentes théories normatives donnent au domaine de l'éthique, et plus précisément l'importance qu'elles accordent au rapport à soi-même. La distinction entre morales maximaliste et minimaliste repose dès lors sur un principe central, celui de l'asymétrie morale entre le rapport à soi et le rapport aux autres. Ogien défend ainsi l'idée d'une éthique minimale qui «nous demande d'éviter de juger "moral" ou "immoral" tout ce qui, dans nos façons de vivre ou nos actions ne concerne que nous-mêmes » $(2007: 197)$. Dans cette perspective, il ne s'agit pas de régenter tous les aspects de notre vie, ni de promouvoir une conception particulière de la vie réussie, mais d'affirmer des principes élémentaires de coexistence des libertés individuelles. 
7 En étendant le principe de liberté aux relations privées entre personnes, l'éthique minimale formalisée par Ogien se donne pour objectif de combattre le maximalisme moral, c'est-à-dire une conception intrusive de la morale qui se manifeste sous trois formes conjointes (Ogien, 2007 : 122-143) : le moralisme, qui veut nous convertir à ses choix au nom de principes dominants dans une société particulière ; le paternalisme, qui nous pousse à vouloir décider pour les autres ce qui est bon pour eux au nom de grands principes tels la dignité ou le respect de la nature humaine ; et le perfectionnisme, qui prétend détenir les critères de l'excellence humaine.

\section{Recension des études empiriques}

8 Si la distinction opérée entre le minimalisme et le maximalisme appelle la question normative de savoir s'il faut être maximaliste ou minimaliste, elle soulève aussi la question empirique de savoir si l'on est maximaliste ou minimaliste. Mill, dont les travaux ont contribué au développement des théories minimales en éthique, a formulé une réponse plutôt tranchée, définie par Ogien comme l'« hypothèse de Mill » :

Il n'est pas difficile de montrer, par de nombreux exemples, qu'étendre les limites de ce qu'on peut appeler la police morale, jusqu'à ce qu'elle empiète sur la liberté la plus incontestablement légitime de l'individu, est, de tous les penchants humains, l'un des plus universels. (Mill, 1859 [1990] : 147)

9 Toutefois, comme l'a souligné Ogien (2007), les recherches expérimentales, menées dans une perspective psychologique, n'ont pas permis de proposer une réponse aussi claire et définitive. Les études les plus connues sur cette question, celles de Turiel et de Haidt, s'opposent même assez radicalement dans leur conclusion. Les données obtenues par Turiel (1983) semblent établir que nous serions plutôt minimalistes. À l'opposé, les travaux de Haidt (2001) aboutissent à la conclusion que nos intuitions morales sont plutôt maximalistes. Ainsi, l'hypothèse de Mill est controversée.

En réalité, résume Ogien, les chercheurs sont partagés entre deux hypothèses opposées. Pour les uns, notre morale de base est pauvre, minimale, et il faut un travail social considérable pour faire de nous des moralisateurs intolérants à l'égard des styles de vie différents du nôtre, toujours tentés de mettre notre nez dans les affaires des autres. Pour les autres, notre morale de base est riche et il faut un travail sociable considérable pour faire de nous des libéraux tolérants à l'égard des styles de vie différents du nôtre, et respectueux de l'intimité des autres. (2011:121)

10 À l'évidence, cette question empirique qui préoccupe actuellement la psychologie expérimentale mérite d'être davantage explorée.

11 L'ambition de notre étude est toutefois nettement plus modeste. Il ne s'agit pas ici de déterminer si nous sommes naturellement maximalistes ou minimalistes, mais d'examiner si les enseignants de langue-culture en contexte multiculturel recourent à des repères moraux minimalistes et, le cas échéant, dans quelle mesure ils y recourent. Dans cette perspective, la recherche empirique menée par Lorius (2014) nous offre un premier éclairage.

12 À partir de la distinction entre le minimalisme et le maximalisme, l'auteur a cherché à comprendre comment les éducateurs orientent leur action dans un milieu scolaire marqué par le pluralisme des valeurs. Plus précisément, il a examiné l'hypothèse du recours à une éthique minimale dans les pratiques éducatives scolaires. À travers la mise en évidence de la pluralité des conceptions morales des éducateurs, il est parvenu 
à la conclusion que le minimalisme constitue une orientation possible de l'éthique éducative.

S'appuyant sur ce résultat, notre étude visera donc non seulement à confirmer que le minimalisme est une orientation possible de l'éthique éducative, mais aussi à explorer s'il peut constituer un repère privilégié de l'éthique enseignante en contexte multiculturel.

\section{Méthodologie de l'enquête}

14 Afin de tester notre hypothèse, nous avons choisi de recourir à la méthode d'analyse combinée (Maurer, 2013). Après en avoir présenté succinctement le fondement théorique, nous rendrons compte de la manière dont nous avons élaboré notre questionnaire ainsi que de son mode de passation.

\section{La méthode d'analyse combinée}

15 La méthode d'analyse combinée (désormais MAC) vise à pallier les insuffisances des outils habituellement utilisés dans la compréhension des représentations sociales. Partant du constat, d'une part, que les enquêtes par questionnaires posent des problèmes de fiabilité statistique et, d'autre part, que les enquêtes par entretiens ne permettent pas de procéder à des généralisations, elle propose de dépasser le clivage des approches qualitative et quantitative :

L'outil que nous proposons, écrit Maurer, permet une vision qualitative fondée sur des éléments quantitatifs, il discerne l'importance relative des différents cognèmes d'une représentation sur une base statistique (non fondée sur des calculs de pourcentage) et permet donc d'articuler les approches quantitative et qualitative. $(2011: 181)$

16 La MAC puise ainsi une partie de son fondement théorique dans la psychologique sociale. Dans cette perspective, la notion de "cognème " réfère à l'unité minimale d'une représentation sociale. Basée sur le concept de «noyau central», la MAC vise à identifier le caractère central ou périphérique d'un cognème ou d'un ensemble de cognèmes.

La méthodologie d'enquête, précise Maurer, repose sur une évolution de la théorie du noyau central, élaborée en psychologie sociale, qui suppose que parmi les éléments composant une représentation sociale (les cognèmes), tous ne jouent pas le même rôle, certains appartenant au noyau de la représentation sociale, d'autres à sa " périphérie ». (2011: 181)

17 Afin de discriminer les cognèmes les plus centraux, la MAC procède à une évaluation selon trois dimensions: adhésion, consensus et distance. L'adhésion correspond au degré d'attachement ou de rejet que les sujets d'un groupe manifestent à l'égard d'un cognème. Le consensus permet d'évaluer dans quelle mesure l'adhésion ou le rejet sont partagés par les sujets. La distance correspond au degré de proximité ou d'éloignement des cognèmes entre eux. Le traitement statistique de ces trois dimensions permet d'accéder à l'organisation des représentations sociales.

De manière plus contextualisée, nous recourrons à l'ensemble de ces concepts lors de la présentation des résultats. Le développement suivant, dans lequel nous rendons compte du processus d'élaboration de notre questionnaire, sera aussi l'occasion de 
présenter plus en profondeur la MAC en exposant la manière dont nous l'avons adaptée aux objectifs particuliers de notre recherche.

\section{L'élaboration du questionnaire}

En vue de constituer une liste d'items, la première étape de la MAC consiste à recueillir le discours des sujets d'un groupe à propos d'un objet de représentation. Cherchant à tester l'hypothèse du recours à une éthique minimale, notre recherche possédait une forte intentionnalité; elle s'inscrivait dans un cadre théorique précis. Il ne s'agissait pas seulement de mettre au jour la représentation que les enseignants ont de leur mission, mais aussi de vérifier si cette représentation est plutôt minimaliste ou maximaliste. À cette fin, nous avons élaboré les items de notre questionnaire en nous appuyant, d'une part, sur la distinction entre le minimalisme et le maximalisme et, d'autre part, sur le constat que l'éducation plurilingue et interculturelle (désormais EPI) relève d'une conception maximaliste de la morale. En somme, il s'est agi d'évaluer si la conception que les enquêtés ont de leur mission était en phase avec la conception maximaliste de l'EPI.

Deux questions ont été élaborées, chacune composée d'une liste de 10 items, soit un multiple de 5 , conformément au principe de la MAC. La première question, centrale, consistait à demander aux enquêtés d'effectuer un classement entre un ensemble d'items dont la moitié correspondait au pôle maximaliste et l'autre moitié au pôle minimaliste. Dans le tableau suivant, nous reproduisons les 10 items obtenus.

Tableau 1 - Répartition des items de la question 1 de l'enquête par questionnaires

\begin{tabular}{|c|c|}
\hline Pôles & Items \\
\hline \multirow{5}{*}{+} & inculquer des valeurs, des attitudes et des comportements \\
\hline & promouvoir les droits de l'homme \\
\hline & être un citoyen exemplaire \\
\hline & former des citoyens exemplaires \\
\hline & perfectionner la personnalité et le caractère de ses apprenants \\
\hline \multirow{5}{*}{-} & respecter en toutes circonstances la liberté individuelle de ses apprenants \\
\hline & chercher prioritairement à ne pas nuire à ses apprenants \\
\hline & $\begin{array}{l}\text { rester neutre sur les questions du bien ou de l'épanouissement personnel de ses } \\
\text { apprenants }\end{array}$ \\
\hline & éviter d'enseigner une manière particulière d'être et de penser \\
\hline & $\begin{array}{l}\text { tolérer tous les comportements de ses apprenants du moment qu'ils ne nuisent } \\
\text { pas aux autres }\end{array}$ \\
\hline
\end{tabular}

Le signe positif (+) correspond au pôle maximaliste et le signe négatif (-) au pôle minimaliste.

Ces 10 items ont ensuite été mélangés puis répartis dans un tableau de manière à ce que les enquêtés, après avoir lu l'ensemble des propositions, puissent les classer selon leur degré d'adhésion. Pour plus de clarté, nous reproduisons ici la question 1 telle qu'elle a été administrée aux enquêtés :

Question 1 
Lisez attentivement le début de la phrase et les 10 propositions suivantes. Classez ces propositions de 1 à 10 selon votre degré d'accord (1 étant la proposition âjec laquelle vous êtes le plus en accord).

Pour moi, un enseignant de langue doit...

\begin{tabular}{|l|l|}
\hline Propositions & Classement de 1 à 10 \\
\hline inculquer des valeurs, des attitudes et des comportements. & \\
\hline chercher prioritairement à ne pas nuire à ses apprenants. & \\
\hline être un citoyen exemplaire. & \\
\hline éviter d'enseigner une manière particulière d'être et de penser. & \\
\hline perfectionner la personnalité et le caractère de ses apprenants. & \\
\hline $\begin{array}{l}\text { respecter en toutes circonstances la liberté individuelle de ses } \\
\text { apprenants. }\end{array}$ & \\
\hline promouvoir les droits de l'homme. & \\
\hline $\begin{array}{l}\text { rester neutre sur les questions du bien ou de l'épanouissement } \\
\text { personnel de ses apprenants. }\end{array}$ & \\
\hline $\begin{array}{l}\text { former des citoyens exemplaires. } \\
\text { tolérer tous les comportements de ses apprenants du moment qu'ils } \\
\text { ne nuisent pas aux autres. }\end{array}$ & \\
\hline
\end{tabular}

La seconde question consistait à demander aux enquêtés d'effectuer un classement entre un ensemble d'items correspondant à des savoirs, des savoir-faire ou des savoirêtre. Basée sur le constat selon lequel l'enseignement de savoir-être repose sur une vision maximaliste de l'éthique, cette question visait à évaluer la place que les enquêtés lui accordent. Il s'agissait ici de mettre en place un processus de contrôle de l'enquête en s'assurant que les résultats des deux questions se corroborent mutuellement. Dans le tableau suivant, nous reproduisons les 10 items retenus pour chacune des 3 compétences :

Tableau 2 - Répartition des items de la question 2 de l'enquête par questionnaires

\begin{tabular}{|l|l|}
\hline Types de compétence & Items \\
\hline \multirow{4}{*}{ savoir } & connaissances lexicales \\
\cline { 2 - 3 } & connaissances grammaticales \\
\cline { 2 - 3 } & connaissances culturelles sur la France et la francophonie \\
\hline \multirow{4}{*}{ savoir-faire } & capacité à utiliser le français lors d'un séjour touristique \\
\cline { 2 - 3 } & capacité à exprimer une opinion en français \\
\cline { 2 - 3 } & capacité à communiquer en français \\
\hline \multirow{4}{*}{} & respect de la diversité linguistique et culturelle \\
\cline { 2 - 2 } & capacité à se mettre à la place de l'autre \\
\cline { 2 - 2 } & curiosité envers l'altérité \\
\cline { 2 - 2 } & volonté de relativiser leur propre point de vue \\
\hline
\end{tabular}

Comme pour la première question, les 10 items ont été mélangés puis répartis dans un tableau de manière à ce que les enquêtés opèrent un classement. Pour plus de clarté, nous reproduisons ici la question 2 telle qu'elle a été administrée aux enquêtés.

Question 2 
Lisez attentivement le début de la phrase et les 10 propositions suivantes. Classez ces propositions de 1 à 10 selon votre degré d'accord (1 étant la proposition avec laquelle vous êtes le plus en accord).

En tant qu'enseignant de FLE, je me fixe pour objectifs de développer chez mes apprenants...

\begin{tabular}{|l|l|}
\hline \multicolumn{1}{|c|}{ Propositions } & Classement de 1 à 10 \\
\hline leur capacité à se mettre à la place de l'autre. & \\
\hline $\begin{array}{c}\text { leur capacité à utiliser le français lors d'un séjour } \\
\text { touristique. }\end{array}$ & \\
\hline leurs connaissances lexicales. & \\
\hline leur respect de la diversité linguistique et culturelle. & \\
\hline leur capacité à exprimer une opinion en français. & \\
\hline leurs connaissances grammaticales. & \\
\hline leur curiosité envers l'altérité. & \\
\hline leur capacité à communiquer en français. & \\
\hline leurs connaissances culturelles sur la France et la \\
francophonie.
\end{tabular}

\section{La passation du questionnaire}

Afin de pouvoir obtenir des résultats fiables, une enquête menée selon la MAC doit porter sur un groupe de 20 sujets au minimum. Étant nous-même enseignant de langueculture dans une université japonaise, nous avons décidé, par commodité, de réaliser cette enquête avec des collègues enseignants natifs de FLE à l'université au Japon. Après avoir été testée et affinée, l'enquête a été envoyée en document joint par courriel à un groupe d'une trentaine d'enseignants dont nous avons pu obtenir l'adresse email. Pour faciliter la passation des questionnaires et donner aux enquêtés la possibilité de conserver l'anonymat, nous avons hébergé notre questionnaire sur un site internet. Lors de l'envoi du courriel, nous avons invité les enquêtés à compléter le questionnaire en ligne ou, selon leur préférence, à nous le retourner directement par courriel. Après plusieurs rappels, nous avons finalement obtenu 25 questionnaires exploitables, dont 18 ont été remplis en ligne.

\section{Présentation des résultats}

Conformément à la procédure de la MAC, nous avons saisi l'ensemble des données obtenues pour chaque réponse sur une feuille de calcul disponible sur le site de la méthode. Deux graphiques, correspondant aux résultats de nos deux questions, ont été générés automatiquement. Ils donnent à voir, pour chaque item, le score d'adhésion et l'indice de consensus ${ }^{1}$. Le score d'adhésion est indiqué sur l'axe gradué des abscisses. Sur la partie gauche figurent les éléments rejetés par le groupe, et sur la partie droite, ceux qui ont recueilli l'adhésion maximale. L'indice de consensus est représenté par un cercle. Plus le diamètre du cercle est important, plus le consensus est grand. Nous 
rendons compte successivement ici des résultats obtenus pour chaque question en commentant brièvement la représentation graphique correspondante.

\section{Résultats obtenus à la question 1 du questionnaire}

Dans le graphique suivant, la numérotation des items est basée sur l'ordre dans lequel ils ont été proposés aux enquêtés. Les propositions sont abrégées. Par ailleurs, les items qui correspondent au pôle maximaliste sont indiqués par le signe positif (+), et ceux qui correspondent au pôle minimaliste sont indiqués par le signe négatif (-).

Figure 1 - Traitement statistique des données de la question 1 du questionnaire

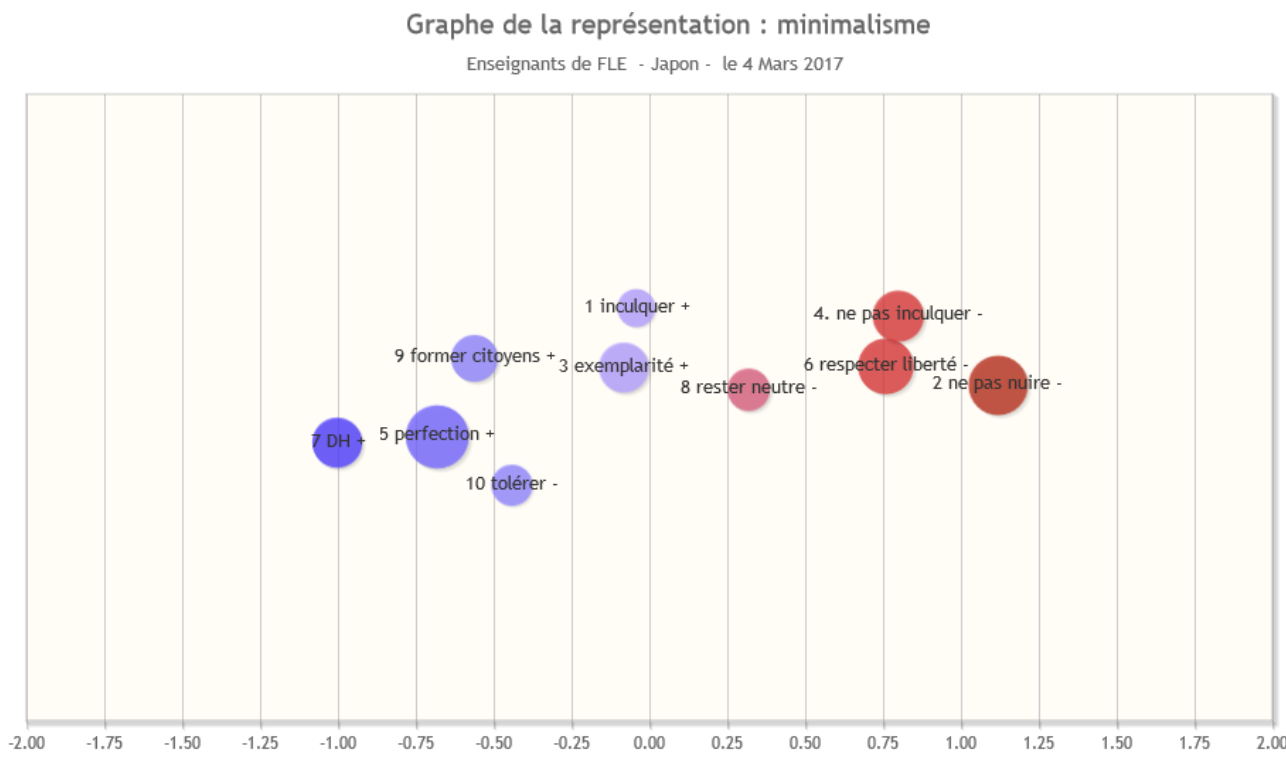

En premier lieu, on constate que les items qui reçoivent la plus forte adhésion, à droite dans le graphique, correspondent au pôle minimaliste. Inversement, les items correspondant au pôle maximaliste font tous l'objet d'un rejet. Sur la base de cette première constatation, nous pouvons valider l'hypothèse selon laquelle notre public d'enquêtés a une conception plutôt minimaliste de la morale. Toutefois, plusieurs indices nous conduisent à nuancer ce résultat :

- Seul l'item 2 «chercher prioritairement à ne pas nuire à ses apprenants » apparaît comme fortement marqué aussi bien du point de vue de l'adhésion que du consensus.

- L'item 10 «tolérer tous les comportements de ses apprenants du moment qu'ils ne nuisent pas aux autres » est rejeté.

- L'item 1 «inculquer des valeurs, des attitudes et des comportements» se situe dans une zone médiane alors qu'il correspond au pôle maximaliste. Nous devons toutefois reconnaitre ici qu'il s'agit d'un item ambigu dans la mesure où il ne donne pas de précision sur le type de valeurs, d'attitudes et de comportements attendus. La portée maximaliste de l'item repose uniquement sur l'emploi du terme "inculquer». Le faible indice de consensus semble exprimer l'ambiguïté de cet item.

- L'item 3 « être un citoyen exemplaire » se situe aussi dans une zone médiane. Il est toutefois intéressant de constater que l'item 9 « former des citoyens exemplaires » est plus fortement rejeté, ce qui est en accord avec le principe minimaliste d'une asymétrie morale entre le rapport à soi et le rapport aux autres. 
D'une manière générale, les scores d'adhésion et de rejet sont relativement faibles. Les parties extrêmes ne sont pas occupées et les items apparaissent comme regroupés.

À défaut de pouvoir valider fermement notre hypothèse, ces résultats nous offrent quelques informations intéressantes sur la représentation que nos enquêtés ont de leur mission éducative au regard des conceptions morales véhiculées par l'EPI. Dans cette perspective, on remarque d'abord que l'item 7 « promouvoir les droits de l'homme » est le plus fortement rejeté par les enquêtés alors que la promotion des droits de l'homme se situe au centre du projet de l'EPI prônée par le Conseil de l'Europe. On remarque ensuite que l'item 4 " éviter d'enseigner une manière particulière d'être et de penser " recueille l'adhésion et que, de manière concomitante, l'item 5 «perfectionner la personnalité et le caractère de ses apprenants " est rejeté par les enquêtés. Dans la mesure où ces 2 items réfèrent assez directement au projet d'enseigner des savoir-être, il convient de vérifier à présent si ces résultats sont corroborés par ceux de la question 2.

\section{Résultats obtenus à la question 2 du questionnaire}

Comme pour la question 1, la numérotation des items est basée sur l'ordre dans lequel ils ont été proposés aux enquêtés. Les propositions sont abrégées. Par ailleurs, les items qui correspondent à des savoirs sont suivis de la lettre $S$; ceux qui correspondent à des savoir-faire sont suivis des lettres SF ; et ceux qui correspondent à des savoir-être sont suivis des lettres $\mathrm{SE}$.

Figure 2 - Traitement statistique des données de la question 2 du questionnaire 
Graphe de la représentation : compétences

Enseignants de FLE - Japon - le 4 Mars 2017
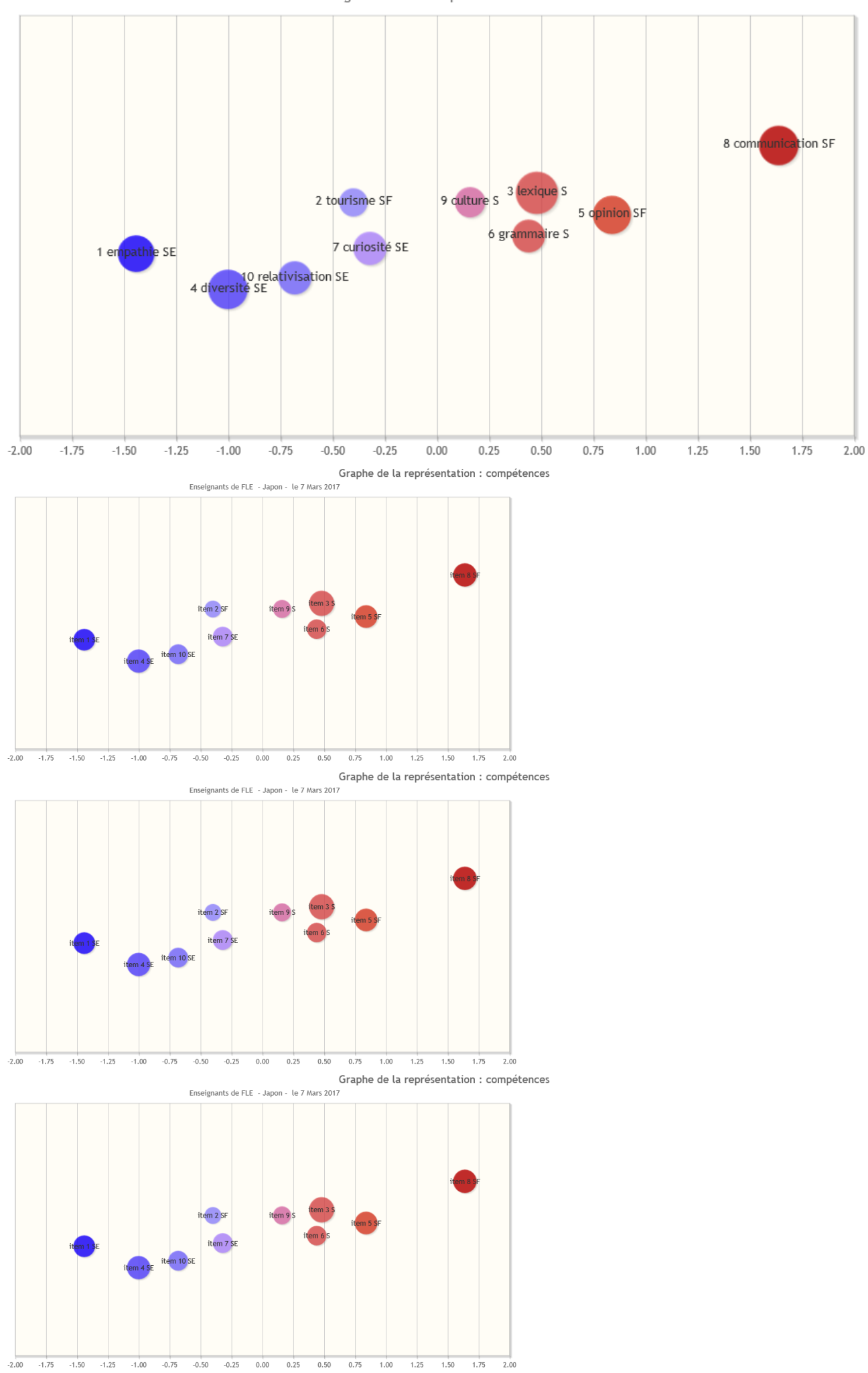

31 En premier lieu, on constate que ce graphique donne une image plus étendue que le graphique précédent. Les scores d'adhésion et de rejet sont plus importants. La taille des cercles montre aussi que le consensus obtenu pour certains items est fort. Plus précisément, on observe que les items correspondant aux savoir-être font tous l'objet 
d'un rejet. L'item 1 «capacité à se mettre à la place de l'autre » et l'item 4 « respect de la diversité linguistique et culturelle » font à la fois l'objet d'un net rejet et d'un fort consensus parmi les enquêtés. Dans une moindre mesure, l'item 10 "volonté de relativiser leur propre point de vue » et l'item 7 « curiosité envers l'altérité » ont aussi été rejetés.

Ces résultats corroborent assez fortement ceux obtenus pour les items 4 et 5 de la question 1. Ils montrent que les enquêtés ne se donnent pas prioritairement comme objectif de "perfectionner la personnalité et le caractère de leurs apprenants ». Si l'on admet par ailleurs l'idée que l'enseignement des savoir-être relève d'une vision maximaliste, on peut considérer que ces résultats sont en accord avec l'hypothèse selon laquelle les enquêtés mobilisent une conception plutôt minimaliste de la morale. À tout le moins, ils ne la contredisent pas.

De nouveau, il est intéressant de mettre ces résultats en regard du projet de l'EPI qui, comme l'a souligné Maurer (2011), tend à accorder une place importante aux savoirêtre au détriment des savoir-faire et des savoirs. Dans cette perspective, on constate d'abord que les 3 items correspondant aux savoirs recueillent l'adhésion des enquêtés. L'item 3 « connaissance lexicale » fait même l'objet d'une adhésion fortement partagée par les enquêtés. On observe ensuite que les deux items recueillant la plus forte adhésion, à droite sur le graphique, sont des items correspondant à des savoir-faire. L'item 8 « capacité à communiquer en français » fait l'objet de la plus forte adhésion.

34 Au total, les résultats obtenus aux 2 questions de notre questionnaire montrent que la conception que les enquêtés ont de leur mission diffère assez nettement de la conception véhiculée par l'EPI. Sur le plan de la morale, les enquêtés mobilisent une conception plutôt minimaliste, laquelle entre en opposition avec le maximalisme caractéristique des travaux du Conseil de l'Europe.

\section{Discussion des résultats}

Compte-tenu du caractère exploratoire de notre étude et de l'absence de recherches empiriques antérieures sur l'éthique professionnelle des enseignants de langue-culture, il nous est difficile de mettre en relation les résultats obtenus ici avec d'autres travaux menés en didactique des langues-cultures. Comme nous l'avons mentionné dans la section consacrée ici-même à la recension des écrits, l'étude menée par Lorius (2014) en sciences de l'éducation nous offre sans doute le meilleur point d'appui.

Ainsi, l'hypothèse selon laquelle le minimalisme constitue une orientation possible de l'éthique éducative, validée par la recherche empirique de Lorius, se trouve aussi confirmée par notre étude. Plus généralement, Lorius a développé l'idée que pour éduquer dans un monde pluraliste, les éducateurs ont besoin d'être eux-mêmes pluralistes, c'est-à-dire de recourir à des repères moraux variés dont certains relèvent du minimalisme. Cette idée nous semble pertinente pour expliquer les résultats obtenus dans notre étude qui montrent que le minimalisme est non seulement une orientation possible mais aussi privilégiée par les enquêtés.

De ce point de vue, la variable contextuelle semble déterminante. Nous avons ciblé ici un public d'enseignants natifs de FLE dans un contexte multiculturel, lequel, par définition, est fortement marqué par le pluralisme des valeurs. Dans un tel contexte, le recours au minimalisme pourrait dès lors être interprété comme un choix prudentiel, 
comme la volonté de s'adapter à une culture éducative, comme le refus ou l'impossibilité d'imposer une conception particulière du bien personnel. En somme, il s'agirait d'un positionnement pragmatique.

De la sorte, il semble important de circonscrire les résultats de notre étude au contexte multiculturel duquel ils sont issus. Il se peut que dans d'autres contextes le minimalisme soit une orientation possible mais non privilégiée par les enseignants. Toutefois, même dans ce cas, il nous semble que l'hypothèse d'une incompatibilité entre les repères moraux mobilisés par les enseignants dans leur pratique et ceux mobilisés par les experts du Conseil de l'Europe dans leur discours devrait être mieux examinée par les promoteurs de l'EPI en didactique des langues-cultures.

$\mathrm{Au}$ minimum, les limites de cette éducation basée sur l'enseignement de savoir-être, c'est-à-dire sur la modification du rapport des élèves à eux-mêmes, devraient être plus clairement prises en compte, en admettant notamment l'idée que dans certains cas, certaines situations, l'orientation perfectionniste de l'EPI risque de déroger au principe de non-nuisance à autrui. Nous rejoignons ici le point de vue de Lorius (2014) qui fait remarquer qu'une approche minimaliste "peut présider à une autolimitation de son action du meilleur aloi lorsque l'on se trouve en situation d'aller au-delà des limites de ses compétences » (p. 100).

\section{Pour ne pas conclure}

Dans cet article, sur la base d'une enquête par questionnaires, nous avons cherché à tester l'hypothèse selon laquelle les enseignants de langue-culture en contexte multiculturel ont une conception plutôt minimaliste de la morale. L'idée de cette recherche est née du constat que l'EPI, qui vise notamment à définir la "posture éthique de chaque enseignant " (Conseil de l'Europe, 2009 : 8), relève d'un maximalisme moral. À travers cette étude, nous avons donc souhaité mettre en regard les repères moraux que mobilisent les enseignants dans leur pratique et ceux que mobilisent les promoteurs de l'EPI en didactique des langues-cultures.

41 Les résultats obtenus à partir du traitement statistique des données de notre questionnaire ont globalement permis de valider notre hypothèse et, ce faisant, de mettre en évidence le décalage entre les conceptions morales de nos enquêtés et celles des promoteurs de l'EPI. En référence aux travaux de Vincent Lorius, nous avons alors développé l'idée que, dans un contexte marqué par le pluralisme moral, le recours au minimalisme apparaît comme une réponse pragmatique, soucieuse de ne pas nuire à autrui.

Si cette étude nous a permis de mieux cerner les conceptions morales des enseignants de langue-culture en contexte multiculturel, il nous semble toutefois important d'en souligner quelques limites. Tout d'abord, nous l'avons dit, notre hypothèse n'a pas été fermement validée. Ainsi, il conviendrait de continuer de la vérifier à l'aide d'un autre dispositif de recherche. La méthode des "expériences de pensée » utilisée en psychologie morale expérimentale - qui consiste à présenter une situation " problématique » à un groupe d'enquêtés et à leur demander ce qu'ils feraient dans une telle situation - pourrait, par exemple, nous permettre d'obtenir des données pertinentes sur les repères moraux mobilisés par les enseignants. 
43 Par ailleurs, notre étude ne concerne qu'un seul public d'enquêtés. Même si le lien entre le pluralisme moral et l'éthique minimale nous donne des raisons de penser que des résultats sensiblement équivalents pourraient être obtenus dans d'autres contextes, l'hypothèse reste à vérifier. De plus, notre enquête porte sur un public d'enseignants à l'université. Il est probable que les repères moraux mobilisés par les enseignants diffèrent en fonction de l'âge des apprenants et du type d'institution éducative. De la sorte, les résultats de cette recherche gagneraient à être mis en comparaison avec ceux d'enquêtes menées auprès de publics différents. Il est à noter que la MAC est précisément destinée à des études comparatives. Dans cette perspective, le questionnaire que nous avons élaboré pourrait servir de point de départ à des recherches futures.

Précisons toutefois que si notre recherche mérite d'être développée, les conclusions obtenues dans une perspective empirique doivent être distinguées de celles obtenues dans une perspective normative. En éthique normative, on pose généralement la distinction entre ce qui est et ce qui doit être, en considérant que de ce qui est on ne peut pas nécessairement dériver ce qui doit être. Il nous semble que ce principe est aussi valable en éthique appliquée. Ainsi, même s'il était clairement établi que les enseignants mobilisent des conceptions plutôt minimalistes, nous ne pourrions en conclure, sans autre argument, que le minimalisme constitue la meilleure option de l'éthique professionnelle des enseignants de langue-culture. C'est en partie pour cette raison que nous avons essayé de développer l'idée, normative, que la reconnaissance du pluralisme moral invalide le projet d'une éducation basée sur le perfectionnement individuel.

Par ailleurs, et nous finirons sur cette idée, si l'état actuel de la recherche empirique ne permet pas d'affirmer que l'éthique enseignante en contexte multiculturel est plutôt minimaliste, l'approche minimaliste constitue, on l'a vu, une orientation possible de l'éthique enseignante. Il nous semble qu'un tel constat devrait déjà interpeller les promoteurs d'une éducation basée sur l'enseignement de savoir-être. Comment justifier, d'un point de vue normatif, un positionnement maximaliste des enseignants ? Comment défendre, dans un monde marqué par le pluralisme, l'idée d'un perfectionnisme moral ? Comment promouvoir, sans prendre le risque de nuire à autrui, une éducation basée sur la modification du rapport des élèves à eux-mêmes? Voilà, entre autres, quelques questions que soulève le projet d'appliquer l'EPI à l'éthique des enseignants de langue-culture. Sans doute ces questions ne sont-elles pas tout à fait au centre des préoccupations des experts du Conseil de l'Europe. Pourtant, elles nous semblent mériter leur examen. C'est en tout cas la moindre des demandes que l'on puisse formuler à des experts qui, ouvertement, nourrissent le projet de définir « la posture éthique » des enseignants de langue-culture. 


\section{BIBLIOGRAPHIE}

XAntier, E. (2017). La problématique éthique du « savoir-être » en didactique des languescultures : quelques réflexions autour du CARAP et autres productions du Conseil de l'Europe, Revue TDFLE, $\mathrm{n}^{\circ} 70$ [en ligne].

Conseil de l'Europe. (2009). L'éducation plurilingue et interculturelle comme projet. Strasbourg : Conseil de l'Europe.

Conseil de l'Europe. (2013). Le CARAP : Un Cadre de Référence pour les Approches Plurielles des Langues et des Cultures : Compétences et ressources. Strasbourg : Conseil de l'Europe.

Conseil de l'Europe. (2001). Un cadre européen commun de référence pour les langues : apprendre, enseigner, évaluer : apprentissage des langues et citoyenneté européenne. Paris : Didier.

Haidt, J. (2001). The Emotional Dog and Its Rational Tail. Psychological Review, vol. 108, n 4, pp. 814-834.

Lorius, V. (2014). Éduquer dans un monde pluraliste. Le minimalisme moral au secours de l'école républicaine? Recherches en Éducation, $n^{\circ}$ 6, pp. 91-102.

Maurer, B. (2011). Méthodologie d'enquête pour une représentation graphique des composants de la représentation sociale d'une langue. In P. Blanchet \& P. Chardenet, Guide pour la recherche en didactique des langues et des cultures une approche contextualisée. Paris : Archives Contemporaines.

Maurer, B. (2013). Représentations Sociales des Langues en Situation Multilingue. Paris : Archives Contemporaines.

Mill, J. S. (1990). De la liberté. Paris : Gallimard.

Ogien, R. (2007). L'éthique aujourd'hui : maximalistes et minimalistes. Paris : Gallimard.

Ogien, R. (2011). L'influence de l'odeur des croissants chauds sur la bonté humaine : et autres questions de philosophie morale expérimentale. Paris : Grasset.

Turiel, E. (1983). The Development of Social Knowledge: Morality and Convention. Cambridge : Cambridge University Press.

\section{NOTES}

1. Dans le cadre de notre étude, le traitement de la distance n'est pas apparu comme pertinent dans la mesure où les items ont été obtenus à l'issue d'un travail d'analyse comportant déjà une recherche intentionnelle de « proximité ».

\section{RÉSUMÉS}

Partant du constat selon lequel l'éducation plurilingue et interculturelle promue par le Conseil de l'Europe repose sur une conception maximaliste de l'éthique, nous nous proposons d'interroger 
les conceptions morales des enseignants de langue-culture en contexte multiculturel. Sont-elles maximalistes ou minimalistes? L'analyse d'une enquête avec des enseignants natifs de FLE à l'université au Japon montre que les enquêtés mobilisent une conception plutôt minimaliste de la morale. Sur la base de ce résultat, nous en appelons à une prise en compte des limites contextuelles de l'éducation plurilingue et interculturelle.

Based on the observation that the plurilingual and intercultural education promoted by the Council of Europe represents a maximalist conception of morality, this paper aims to explore the moral conceptions of teachers of language and culture in a multicultural context. The survey analysis shows that the respondents mobilize a rather minimalist conception of morality. Based on this result, we call for the contextual limitations of plurilingual and intercultural education to be taken into consideration.

\section{INDEX}

Thèmes : Jeunes chercheur.e.s

Keywords : plurilingual and intercultural education, maximalism and minimalism, teacher ethics, multicultural context.

Mots-clés : éducation plurilingue et interculturelle, maximalisme et minimalisme, éthique enseignante, contexte multiculturel d'enseignement-apprentissage.

\section{AUTEUR}

\section{EMMANUEL ANTIER}

Emmanuel Antier a soutenu sa thèse en septembre 2017 sous la direction de Bruno Maurer, Université Paul-Valery Montpellier 3. Il est actuellement Maître de Conférences en Didactique des langues-cultures au département de langue et littérature françaises de l'Université de Fukuoka (Japon). Ses recherches portent principalement sur l'éthique professionnelle des enseignants de langue-culture en contexte multiculturel.

antier[at]fukuoka-u.ac.jp 